\title{
Azimuthal distributions in unpolarized SIDIS
}

\author{
Nathan Harrison \\ Jefferson $L a b$ \\ E-mail: nathanh@jlab.org \\ Harut Avakian* \\ Jefferson Lab \\ E-mail: avakianejlab.org
}

\section{Kyungseon Joo}

University of Connecticut

E-mail: kjoo@jlab.org

\begin{abstract}
We present studies of multiplicities and the $\cos \phi$, and $\cos 2 \phi$ moments, in charged pion production in semi-inclusive deep inelastic scattering (SIDIS). The data set used was the E1-f run from the CEBAF Large Acceptance Spectrometer (CLAS) at Jefferson Lab which ran in 2003. The run used a $5.498 \mathrm{GeV}$ longitudinally polarized electron beam and an unpolarized liquid hydrogen target. Two pion channels $\left(\pi^{+}\right.$and $\left.\pi^{-}\right)$were studied over a broad kinematical range $(x=0.1-0.6$, $Q^{2}=1.0-4.5 \mathrm{GeV}^{2}, z=0.0-1.0, P_{h \perp}^{2}=0.0-1.0 \mathrm{GeV}^{2}$, and $-180^{\circ}<\phi_{h}<180^{\circ}$ ). These measurements give insights into the transverse momentum dependence of parton distribution functions (PDFs) describing the dynamics of quarks and gluons inside of the proton.
\end{abstract}

XXIV International Workshop on Deep-Inelastic Scattering and Related Subjects

11-15 April, 2016

DESY Hamburg, Germany

\footnotetext{
* Speaker.
} 
Semi-inclusive deep-inelastic scattering (SIDIS) has emerged as a powerful tool to probe nucleon structure and to provide access to Transverse Momentum Dependent (TMD) partonic distributions through measurements of spin and azimuthal asymmetries. A SIDIS $(\ell(k)+N(P) \rightarrow$ $\left.\ell^{\prime}\left(k^{\prime}\right)+h\left(P_{h}\right)+X\left(P_{X}\right)\right)$ reaction is one in which a beam lepton, $\ell$ with the 4-momenta $k$, scatters off of a target nucleon, $N$, via the exchange of a photon and the scattered lepton, $\ell^{\prime}$, is detected along with a single hadron, $h$ with 4-momenta $P_{h}$; everything else in the final state, $X$, is ignored.

Assuming single photon exchange, the leptoproduction cross-section for an unpolarized beam and unpolarized target can be written as a sum of structure function (see [1]):

$$
\frac{d^{5} \sigma}{d x d Q^{2} d z d \phi_{h} d P_{h \perp}^{2}} \propto F_{U U, T}+\varepsilon F_{U U, L}+\sqrt{2 \varepsilon(1+\varepsilon)} \cos \phi_{h} F_{U U}^{\cos \phi_{h}}+\varepsilon \cos \left(2 \phi_{h}\right) F_{U U}^{\cos 2 \phi_{h}} .
$$

where structure functions $F_{\ldots} \ldots$ [1] depend on kinematical variables $x, Q^{2}, z, P_{h \perp}$ define the modulations of corresponding azimuthal moments, with azimuthal angle $\phi_{h}$, which is defined between the lepton plane and the hadron production plane. The kinematic variables $x, y$, and $z$ are defined as: $x=Q^{2} / 2(P \cdot q), y=(P \cdot q) /(P \cdot k), z=\left(P_{h} \cdot P\right) /(P \cdot q)$, where $Q^{2}=-q^{2}=-\left(k-k^{\prime}\right)^{2}$ is the fourmomentum of the virtual photon, $\gamma=2 M x / Q, M$ and $M_{h}$ are the nucleon and hadron masses, $P_{h \perp}$ is the transverse momentum of the detected hadron. The ratio $\varepsilon$ of the longitudinal and transverse photon flux is given by: $\varepsilon=\frac{1-y-\gamma^{2} y^{2} / 4}{1-y+y^{2} / 2+\gamma^{2} y^{2} / 4}$.

The final-state gluon radiation in SIDIS was predicted to lead to observable $\cos \phi$ modulations of the SIDIS cross section, and that effect has been proposed by Georgi and Politzer in 1977 [2] as a clean test of $\mathrm{pQCD}$. Perturbative-QCD effects, like gluon radiation, can indeed lead to azimuthal dependencies in the semi-inclusive DIS cross section, but they contribute mainly at large values of $P_{h \perp}$, and are next-to-leading order in the strong coupling constant.

In 1978, Cahn [3] discussed similar asymmetries arising from non-zero intrinsic transverse momenta of partons. Although, suppressed by $P_{h \perp} / Q$ that modulation (known as the Cahn effect), appeared to be significant and dominating in the $P_{h \perp} \sim 1 \mathrm{GeV}$ range. Significant cosine modulations with azimuthal angle of final state hadrons with respect to the lepton scattering plane, $\phi_{h}$, observed in various experiments indicate that higher twist effects can be very important. Additional contribution to $\cos \phi_{h}$ and $\cos 2 \phi_{h}$ moments coming from processes when the final meson is produced at short distances via hard-gluon exchange [4] may also be significant in the kinematic regime where the ejected meson carries most of the virtual photon momentum. It appeared that the interplay between the parton transverse momentum and spin (Boer-Mulders effect [5]) can generate sub leading contribution to $\cos \phi$ and leading-twist contribution to the $\cos 2 \phi$ modulation, which is one the main focuses of our studies.

The structure function $F_{U U, T}$ defining the $\phi$-integrated cross sections could be presented as a convolution of parton distribution function (PDF), $f_{1}$, and fragmentation function (FF), $D_{1}$, which are the usual unpolarized twist-2 PDF and FF, respectively. The structure function $F_{U U}^{\cos 2 \phi_{h}}$ at leading twist can be presented as a convolution of Boer-Mulders distribution function, $h_{1}^{\perp}$ ([5] describing the correlation between the transverse motion of a quark and its own transverse spin, and the Collins fragmentation function, $H_{1}^{\perp}$ [6], describing fragmentation of transversely polarized quarks. The structure function $F_{U U}^{\cos \phi_{h}}$ receives contributions from the convolution of twist-2 and twist-3 distribution and fragmentation functions, such as the twist-3 $h$ related to Boer-Mulders DF $h_{1}^{\perp}$ ([5, 7]), the Collins FF $H_{1}^{\perp}$, and the twist-3 DFs $h$ and $f^{\perp}$ [1]. The higher-twist observables are a 
key for understanding long-range quark-gluon dynamics. They have also been interpreted in terms of average transverse forces acting on a quark at the instant after absorbing the virtual photon [8].

Among the various twist- 3 contributions suppressed as $1 / Q$, several involve either a distribution or fragmentation function that relates to quark-gluon-quark correlations, and hence is interaction dependent and has no probabilistic interpretation. In the Wandzura-Wilczeck approximation [9] all these terms are neglected, and only two contributions are considered:

$$
F_{U U}^{\cos \phi} \simeq-\frac{2 M}{Q} C\left[\frac{\vec{P}_{h \perp} \cdot \vec{k}_{\perp}}{M P_{h \perp}} x f^{\perp} D_{1}+\frac{\vec{P}_{h \perp} \cdot \vec{p}_{\perp}}{z M_{h} P_{h \perp}} x h H_{1}^{\perp}\right]
$$

where the first (second) term is related to the Cahn (Boer-Mulders) effect and $C$ stands for convolution [1]. Several measurements of cosine modulations in semi-inclusive DIS experiments have been published in the past $[10,11,12,13]$. Most measurements averaged over any possible flavour dependence as they refer to hadrons without type nor charge distinction, and only to hydrogen target or hydrogen and deuterium targets combined together.

During the last few years several precise SIDIS measurements have become available. The CLAS collaboration measured non-zero cosine modulations for positive pions produced by $6 \mathrm{GeV} / \mathrm{c}$ electrons scattering off the proton [14]. The HERMES experiment have measured cosine modulations of hadrons produced in the scattering of $27.5 \mathrm{GeV} / \mathrm{c}$ electrons and positrons off pure hydrogen and deuterium targets, where the lepton beam scatters directly off neutrons and protons (with only negligible nuclear effects in case of deuterium) [15]. For the first time these modulations were determined in a four-dimensional kinematic space for positively and negatively charged pions and kaons separately, as well as for unidentified hadrons. At COMPASS, positive and negative hadrons produced by the $160 \mathrm{GeV} / \mathrm{c}$ muon beam scattering off a ${ }^{6} \mathrm{LiD}$ target have been measured in a threedimensional grid of the relevant kinematic variables $x, z$ and $P_{h \perp}^{2}$ [16]. In all the experiments, the new data confirm the existence of a sizable $\cos \phi$ and a non-zero $\cos 2 \phi$ modulations.

The azimuthal modulations have been studied phenomenologically in detail, also using phase space limitations due to finite beam energies of real experiments [17]. The cosine modulation in particular is most sensitive to transverse momentum distributions leading to significant corrections due to limitation of the phase space in experiments. Studies of transverse momentum distributions using the SIDIS multiplicities [18] indicate wide spread of the values for average transverse momentum of quarks due to experimental errors.

This contribution reports measurements of structure functions for the unpolarized beam and target using charged pions from SIDIS from the E1-f CLAS data set using a $5.498 \mathrm{GeV}$ electron beam and the CEBAF Large Acceptance Spectrometer (CLAS) [19] at Jefferson Laboratory. Longitudinally polarized electrons were scattered off an unpolarized liquid-hydrogen target. Scattered electrons were detected in CLAS. Electron candidates were selected by a hardware trigger using a coincidence of the gas Cherenkov counters and the lead-scintillator electromagnetic calorimeter.

Deep-inelastic scattering events were selected by requiring $Q^{2}>1 \mathrm{GeV}^{2}$ and $W>2.05 \mathrm{GeV}$, where $W$ is the invariant mass of the hadronic final state. A minimum value for the $\pi^{ \pm}$transverse momentum, $P_{h \perp}>0.05 \mathrm{GeV}$, ensures that the azimuthal angle $\phi_{h}$ is well-defined. The total number of selected $e \pi^{ \pm}$coincidences was $\approx 6.7 M$ for the presented $z$ range, $0.4<z<0.7$, which selects the 
semi-inclusive region [20]. Events with missing-mass values for the $e \pi^{ \pm} X$ system that are smaller than $1.35 \mathrm{GeV}\left(M_{x}\left(e \pi^{ \pm}\right)<1.35 \mathrm{GeV}\right)$ were discarded to exclude contributions from exclusive processes. This cut helps to remove proton contamination at high momenta in $\pi^{+}$sample, and also cuts out exclusive events $\left(e p \rightarrow e \pi^{+} n\right)$, and the delta resonance.

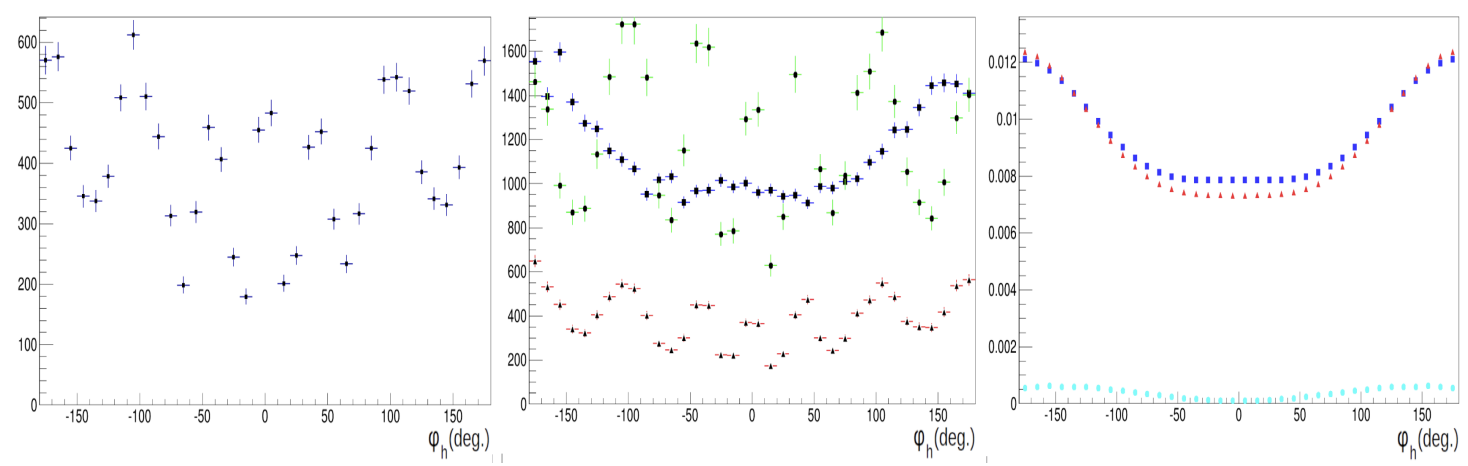

Figure 1: (color online) Dependence on azimuthal angle $\phi_{h}\left(\pi^{+}\right.$channel) of observed counts (left); Monte Carlo (middle) - generated (blue squares), reconstructed (red triangles), and acceptance (green circles) (acceptance scale goes from 0.0 - 0.5); and radiative cross sections (right) - Born cross section (blue squares), radiated cross section (red triangles), and exclusive tail (teal circles), for a given bin in $x, Q^{2}, z$, and $P_{h \perp}^{2}$ $\left(0.2<x<0.3,1.3<Q^{2}<1.7 \mathrm{GeV}^{2}, 0.4<z<0.45,0.4<P_{h \perp}^{2}<0.45 \mathrm{GeV}^{2}\right)$.

A major challenge in extraction of $\phi_{h}$ distributions is the handling of the detector acceptance, both geometrical acceptance (the location of active detector elements) and the efficiency in the active regions. This is done using Monte Carlo simulations. A LUND-MC based event generator (PEPSI [21]) is used to create a realistic large set of events (several iterations may be required before the set is "realistic") which are passed through a GEANT based simulation of the CLAS detector. For a given kinematic space, the acceptance is equal to the number of reconstructed events divided by the number of generated events. The number of events in the E1-f data is then divided by the acceptance to get a corrected value for the number of events. After events have been selected and binned, and after all corrections, including bin centering corrections, have been applied, a table of values of normalized counts in elementary bins has been extracted in 5-dimensional bins in $x, Q^{2}, z, P_{h \perp}$ and $\phi$. The $\phi$-distributions of pions from detector and corresponding MC simulation studies of acceptances are shown in Fig. 1.

HAPRAD version $2.0[22,23]$ was used to calculate radiative corrections in bins by calculating $\sigma_{\text {rad }+ \text { tail }}\left(x, Q^{2}, z, P_{h \perp}^{2}, \phi_{h}\right)$ for a given $\sigma_{B o r n}\left(x, Q^{2}, z, P_{h \perp}^{2}, \phi_{h}\right)$ which is obtained from a model. The radiative correction factor has been used to restore the Born cross-section, $\sigma_{B o r n}=$ $\sigma_{\text {measured }} / R C$ factor. Three different models were used to study model dependence of radiative corrections. Main sources of systematic uncertainties include the acceptance, fitting uncertainties and structure function variations in calculations of radiative corrections.

To extract multiplicities and azimuthal moments, the $\phi_{h}$ distributions for charged pions for each $x-Q^{2}-z-P_{h \perp}^{2}$ bin are fit with the function $a\left(1+b \cos \phi_{h}+c \cos 2 \phi_{h}\right)$. The parameters $a, b$, and $c$ then directly give $A_{0}, A_{U U}^{\cos \phi_{h}}$, and $A_{U U}^{\cos 2 \phi_{h}}$ for each $x-Q^{2}-z-P_{h \perp}^{2}$ bin. A representative plot is shown here in figure 2, which shows $A_{0}, A_{U U}^{\cos \phi_{h}}$, and $A_{U U}^{\cos 2 \phi_{h}}$ vs $P_{h \perp}^{2}$ for one $z$ bin for both pion channels. 

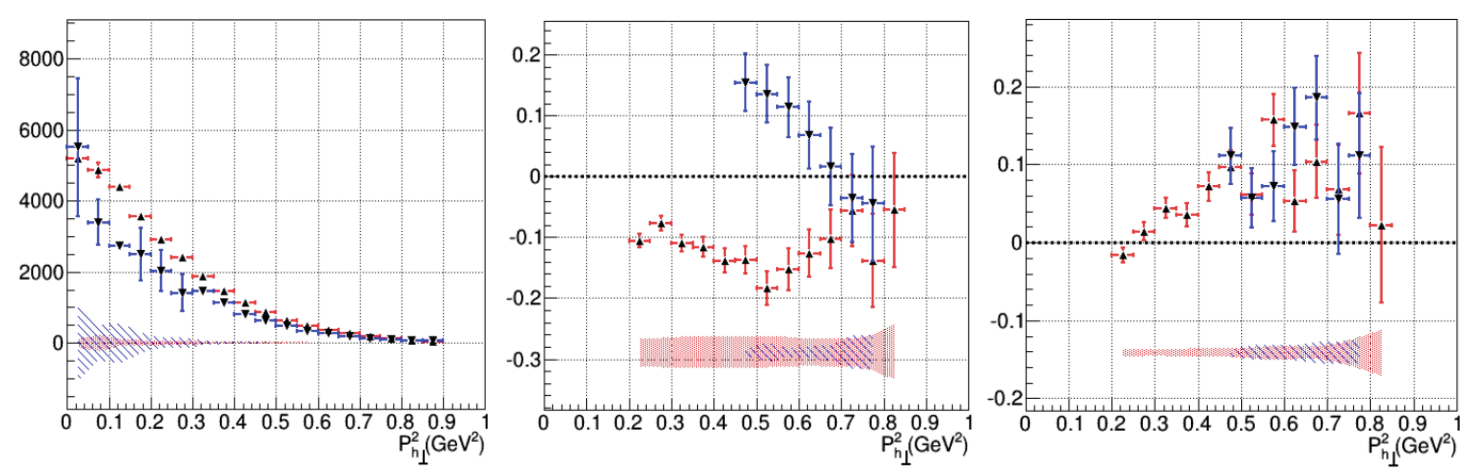

Figure 2: (color online) $A_{0}$ (left), $A_{U U}^{\cos \phi_{h}}$ (middle), and $A_{U U}^{\cos 2 \phi_{h}}$ (right) for the $\pi^{+}$(red upward pointing triangles) and $\pi^{-}$(blue downward pointing triangles) channels as a function of $P_{h \perp}^{2}$ for $0.2<x<0.3$, $0.4<z<0.45$ and $\left\langle Q^{2}\right\rangle \approx 1.95 \mathrm{GeV}^{2}$.

A similar measurement has been published by the CLAS Collaboration using E1-6 data [14]. However, it only measures the $\pi^{+}$channel and has a more limited kinematic range. A comparison for the $\pi^{+}$channel in overlapping kinematic ranges shows good agreement, both qualitatively and quantitatively, implying good consistency between measurements.

The $\cos \phi$ asymmetry gets contributions only at sub-leading twist and can be used to constrain the related terms $[3,24,4]$. The formalism based on the twist-3 approach could be tested in $Q^{2}$ dependence of $\cos \phi$ modulations. In the Fig. 3 CLAS measurements are compared with corresponding measurements from HERMES experiment [15], after taking into account the kinematic factors in the expression of the $\cos \phi$ modulation and $\phi$ independent terms ([1]), with $f(y)=$ $\sqrt{2 \varepsilon(1+\varepsilon)}$. The CLAS and HERMES measurements are found to be consistent with each other in a wide range of $Q^{2}$, as shown in Figs. 3, indicating that at energies as low as 5-6 GeV, the behavior of azimuthal modulations are similar to higher energy measurements. For comparison, the lowest $x$ CLAS bin and highest $x$ HERMES bins were used with equal average value of $x \approx 0.19, z \approx$ 0.45 and $P_{h \perp} \approx 0.42 \mathrm{GeV}^{2}$. The CLAS data provide significant improvements in the precision of azimuthal moments for the kinematic region where the two data sets overlap, and they extend the measurements to the large $x$ region not accessible at HERMES.
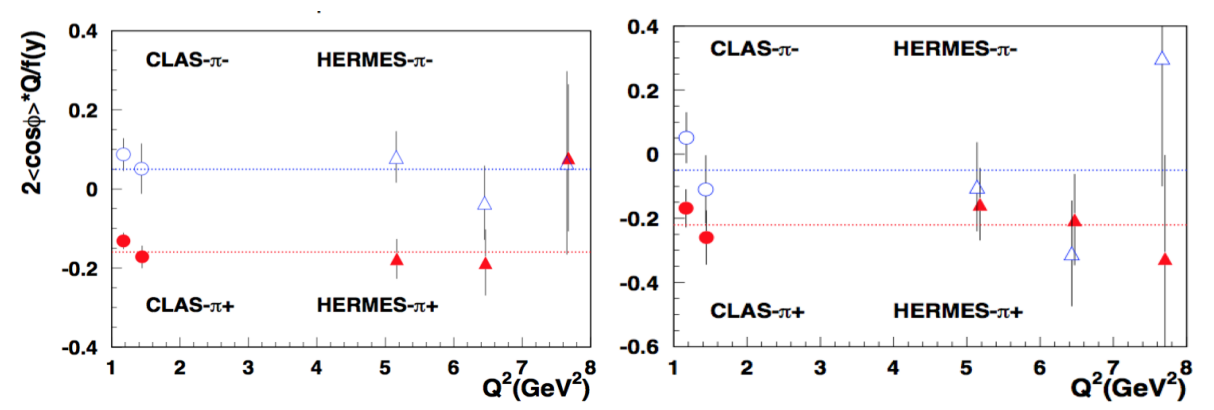

Figure 3: (Color online) The $\cos \phi$ modulations in $\pi^{ \pm}$SIDIS plotted vs $Q^{2}$ for a bins with $x \approx 0.19$, and $z \approx 0.35, P_{h \perp} \approx 0.6 \mathrm{GeV}$ (left) and $z \approx 0.43, P_{h \perp} \approx 0.8 \mathrm{GeV}$ (right). Filled symbols are for $\pi^{+}$and open symbols for $\pi^{-}$. The triangles CLAS and open symbols are for HERMES [15]. 
In summary, we have presented measurements of the kinematic dependences of the azimuthal modulations of in semi-inclusive $\pi^{ \pm}$electroproduction from the E1-f CLAS data set, indicating significant dependence on the flavor of final state. The results for $\cos \phi$-moment are compared with published HERMES data [15], providing a significant improvement in precision and can serve as an important input for studies of higher-twist effects. The $Q^{2}$-dependence of the $\cos \phi$ modulation is consistent with the twist-3 nature of the contribution and within statistical uncertainties is consistent with measurements performed at much higher energies and $Q^{2}$. Higher precision data from $12 \mathrm{GeV}$ running of JLab will be required to study the $Q^{2}$-dependence of observables in more details.

We thank A. Prokudin and Peter Schweitzer for useful and stimulating discussions. This material is based upon work supported by the U.S. Department of Energy, Office of Science, Office of Nuclear Physics under contract DE-AC05-06OR23177.

\section{References}

[1] A. Bacchetta et al., JHEP 02 (2007) 093, hep-ph / 0611265.

[2] H. Georgi and H.D. Politzer, Phys. Rev. Lett. 40 (1978) 3.

[3] R.N. Cahn, Phys. Lett. B78 (1978) 269.

[4] E.L. Berger, Z. Phys. C4 (1980) 289.

[5] D. Boer and P.J. Mulders, Phys. Rev. D57 (1998) 5780, hep-ph / 9711485.

[6] J.C. Collins, Nucl. Phys. B396 (1993) 161, hep-ph / 9208213.

[7] B. Pasquini and F. Yuan, Phys. Rev. D81 (2010) 114013, arXiv: 1001.5398.

[8] M. Burkardt, hep-ph 0807.2599 (2008), arXiv: hep-ph / 0807.2599.

[9] S. Wandzura and F. Wilczek, Phys. Lett. B72 (1977) 195.

[10] European Muon, J.J. Aubert et al., Phys. Lett. B130 (1983) 118.

[11] European Muon, M. Arneodo et al., Z. Phys. C34 (1987) 277.

[12] E665, M.R. Adams et al., Phys. Rev. D48 (1993) 5057.

[13] ZEUS, J. Breitweg et al., Phys. Lett. B481 (2000) 199, hep-ex/ 0003017.

[14] CLAS, M. Osipenko et al., Phys. Rev. D80 (2009) 032004, arXiv: hep-ex/ 0809.1153.

[15] HERMES Collaboration, A. Airapetian et al., Phys.Rev. D87 (2013) 012010, arXiv: 1204.4161.

[16] COMPASS, C. Adolph et al., Nucl. Phys. B886 (2014) 1046, arXiv: 1401.6284.

[17] M. Boglione, S. Melis and A. Prokudin, Phys.Rev. D84 (2011) 034033, arXiv:1106. 6177.

[18] A. Signori et al., JHEP 1311 (2013) 194, arXiv:1309.3507.

[19] CLAS Collaboration, B. Mecking et al., Nucl.Instrum.Meth. A503 (2003) 513.

[20] CLAS Collaboration, H. Avakian et al., Phys. Rev. Lett. 105 (2010) 262002, arXiv:hep-ex/1003.4549.

[21] L. Mankiewicz, A. Schafer and M. Veltri, Comput. Phys. Commun. 71 (1992) 305.

[22] I. Akushevich, N. Shumeiko and A. Soroko, Eur. Phys. J. C10 (1999) 681, hep-ph/ 9903325.

[23] I. Akushevich, A. Ilyichev and M. Osipenko, Phys.Lett. B672 (2009) 35, arXiv: 0711.4789.

[24] M. Anselmino et al., Phys. Rev. D71 (2005) 074006, hep-ph $/ 0501196$. 